\title{
Comment on "Reference Values of Total Lean Mass, Appendicular Lean Mass, and Fat Mass Measured with Dual- Energy X-ray Absorptiometry in a Healthy Mexican Population”
}

\author{
Eva Amado-Bonilla ${ }^{1}$ Maria I. Pintado-Vidal ${ }^{1} \cdot$ Maria I. Mangas-Borrego $^{1} \cdot$ \\ Estefania Marchena-Ortiz ${ }^{1} \cdot$ Jorge Barrantes-Chacon $^{1} \cdot$ Jose M. Moran $^{1}(\mathbb{D}$
}

Received: 9 October 2016/Accepted: 27 October 2016/Published online: 4 November 2016

(C) Springer Science+Business Media New York 2016

We have read with interest the paper from Clark et al. [1] about the establishment of Reference Values of Total Lean Mass, Appendicular Lean Mass, and Fat Mass Measured with Dual-Energy X-ray Absorptiometry in a Healthy Mexican Population. Besides the intrinsic interest of the manuscript itself we would like to make the following comments that although might not affect the overall conclusions of the paper, might affect the data presented and hence the accuracy of the provided reference tables.

In the abstract section it is indicated that a total of 2829 males and 6694 females were included in the final analysis. It comprises a total of 9523 participants that do not agree with the 9518 indicated also in the abstract section. Again, in the description of the study population in the Methods section of the paper a total of 9518 participants (described as 2829 males and 6694 females) were included in the study but as indicated, such distribution comprises 9523 participants. Such participant's distribution agrees with the data showed in the tables of Clark and colleagues paper, which represent the participants grouped by age. Surprisingly such patient distribution does not agree with the data provided by the authors as supplementary material [2]. Such data show the patient distribution by age. There are a total of 2796 males and 6701 females represented in such table that comprises a total population of 9497 participants. If we analyze, for example, the variables distribution in the group of 7-10 years, there are a total of 126 males and 147 females in the supplementary data versus 141 males and 162 females

Jose M. Moran

jmmorang@unex.es

1 Nursing Department, Health Science Research Methodology Collaboration Group, School of Nursing and Occupational Therapy, University of Extremadura, Cáceres, Spain represented in Table 1 and others. Although the participant's distribution does not agree between the tables presented in the manuscript and the supplementary data, there are variables measured in the participants that agree between data groups. For example, the mean of total lean body mass (LBM) for the age group of 7-10 years in the male group is $21.9 \pm 3.8(\mathrm{~kg})($ mean $\pm \mathrm{SD})$ as reported in Table 3; after some algebra calculations and based on the data provided in the supplementary material the mean of LBM for the age group (7-10 years) agrees with the data presented in Table 3 $(21.94 \pm 3.87)(\mathrm{kg})($ mean $\pm \mathrm{SD})$ in the male group. Similarly, data for the female group in such age group (7-10 years) virtually agree between Table 3 of the Clark and colleague's paper and the supplementary data $(21.2 \pm 4.3$ vs. $21.37 \pm 4.29)$. There are slight differences in other groups (i.e., males $50-59$ years, LBM $50.2 \pm 6.1 \mathrm{~kg}$ in Table 3 and $50.85 \pm 6.83 \mathrm{~kg}$ from supplementary data). We think that authors should notice such discrepancies and hence provide an explanation.

Conflict of interest Eva Amado-Bonilla, Maria I. Pintado-Vidal, Maria I. Mangas-Borrego, Estefania Marchena-Ortiz, Jorge Barrantes-Chacon and Jose M. Moran declare that they have no conflict of interest.

\section{References}

1. Clark P, Denova-Gutiérrez E, Ambrosi R et al (2016) Reference values of total lean mass, appendicular lean mass, and fat mass measured with dual-energy X-ray absorptiometry in a healthy Mexican population. Calcif Tissue Int 99:462. doi:10.1007/ s00223-016-0181-z

2. Clark P et al (2016) Supplementary material 1. Calcif Tissue Int. https://static-content.springer.com/esm/art\%3A10.1007\%2Fs00223016-0181-z/MediaObjects/223_2016_181_MOESM1_ESM.docx. Accessed 9 Oct 2016 